\title{
Long term performance of the COSY/Jülich polarized ion source
}

\author{
O. Felden, R. Gebel*, R. Maier, S. Mey \\ Forschungszentrum Jülich Institute for Nuclear Physics (IKP-4) 52425 Jülich, Germany \\ E-mail: r.gebel@fz-juelich.de
}

The COoler SYnchrotron and storage ring COSY at the Forschungszentrum Jülich accelerates protons and deuterons to momenta between $0.6 \mathrm{GeV} / \mathrm{c}$ and $3.8 \mathrm{GeV} / \mathrm{c}$. Polarized $\mathrm{H}^{-}\left(\mathrm{D}^{-}\right)$beams are delivered routinely by the colliding beams source, accelerated in the cyclotron JULIC and injected via stripping injection into the COSY ring since 1996 (2003). In parallel to beam delivery to the synchrotron, the atomic beam part, the cesium ionizer, neutralizer and the ion extraction of the polarized ion source have been optimized for pulsed operation and the number of polarized particles for injection into the cyclotron has reached about $5 \times 10^{12}$ protons, delivered in $20 \mathrm{~ms}$ pulses with repetition rates up to 2 seconds. In order to provide polarized beams with the highest possible intensity and polarization routinely, components of the source and diagnostic tools for neutral beams and polarization measurements have been optimized over the last years. The production process of cesium dispenser ionizers at the Forschungszentrum has been improved substantially and a new generation of porous tungsten dispensers is now available. In parallel laser cleaning has been applied and improved the production process, the start performance significantly and allowed also recovery of used dispensers.

PACS: 29.25.Lg, 29.25.Ni, 29.27.Hj, 29.20.D-, 29.20.db, 29.20.dk

XVth International Workshop on Polarized Sources, Targets, and Polarimetry,

September 9-13, 2013

Charlottesville, Virginia, USA

${ }^{*}$ Speaker. 


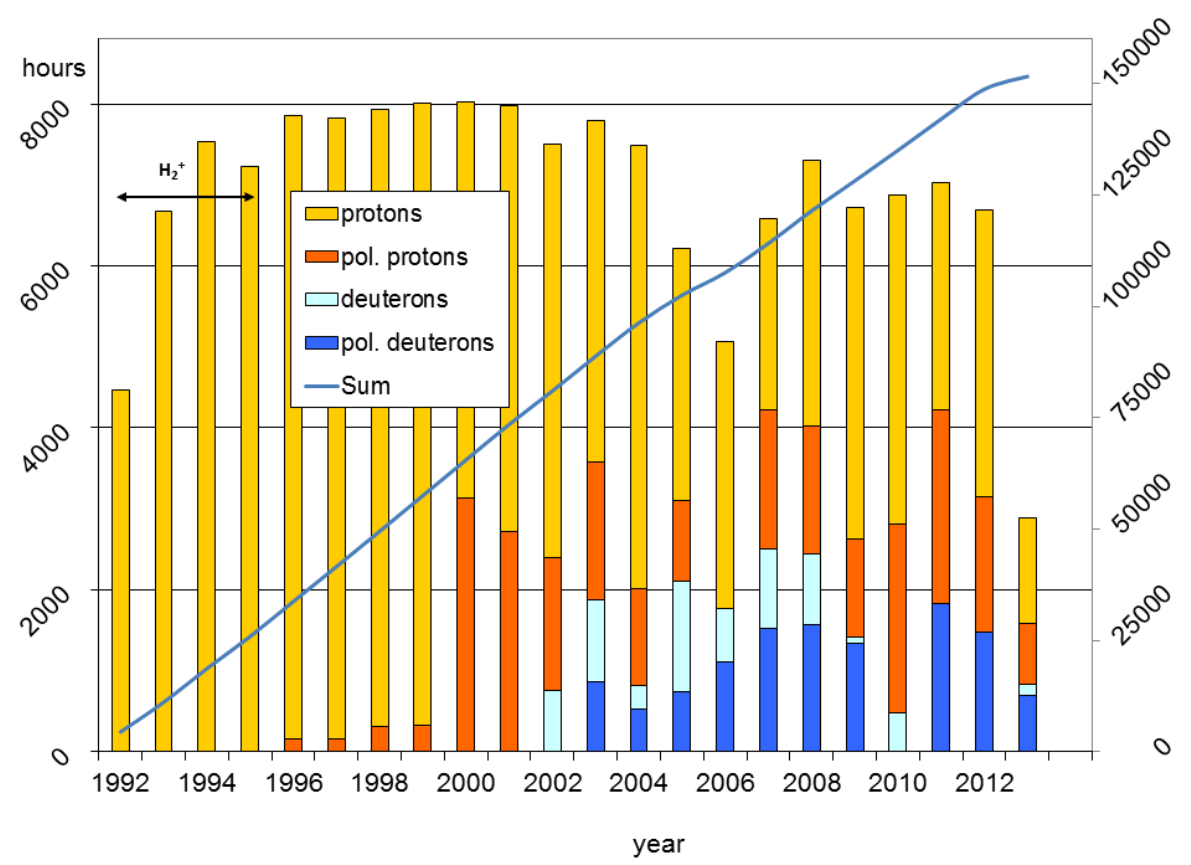

Figure 1: Cosy and injector statistics: operational hours and scheduled beam species.

\section{Introduction}

The Nuclear Physics Institute (IKP), as a member of the Jülich Centre for Hadron Physics (JCHP), is dedicated to fundamental research in the field of hadron, particle, and nuclear physics. The IKP is a department located at the Forschungszentrum Jülich[1], one of the largest members of the Helmholtz Association (HGF). COSY[2] is a cooler synchrotron and storage ring for light ions routinely providing protons and deuterons in the momentum range between 600 and 3700 $\mathrm{MeV} / \mathrm{c}$, corresponding to 175 and $2880 \mathrm{MeV}$ energy. Beams with the desired energy are available for experiments with the circulating beam for two internal experiments - ANKE and WASA - as well as for one experiment with the extracted beam - TOF - at an external target position. All three detection systems are operated by large international collaborations. In addition, the unique COSY capabilities are used by the SPIN@COSY-, dEDM-, JEDI and PAX-collaborations[1] to investigate spin-manipulations. PAX prepares experiments on polarization buildup in storage rings. The srEDM and JEDI collaborations study spin manipulations as preparation to build dedicated storage rings to search for electric dipole moments of light ions ( $\mathrm{p}, \mathrm{d},{ }^{3} \mathrm{He}$ ), and intends using COSY as an injector for such a ring. The HESR synchrotron, part of the international GSI project Facility for Antiproton and Ion Research (FAIR) [3], is dedicated to the field of high energy antiproton physics with high quality beams over the broad momentum range from 1.5 to $15 \mathrm{GeV} / \mathrm{c}$ to explore the research areas of hadron structure and quark-gluon dynamics. In the upcoming decade, the IKP branch of hadron physics will undergo a transition from light quark systems to hadrons with charm quarks, which will be pursued at FAIR. 


\begin{tabular}{|r|r|r|r|r|}
\hline & protons & deuterons & pol. protons & pol. deuterons \\
\hline beam hours & 52800 & 6600 & 23000 & 11700 \\
\hline fraction & $56 \%$ & $7 \%$ & $24 \%$ & $12 \%$ \\
\hline sum & unpol. & $59400 \mathrm{~h}$ & pol. & $34700 \mathrm{~h}$ \\
\hline average & & $4570 \mathrm{~h} / \mathrm{y}$ & & $2670 \mathrm{~h} / \mathrm{y}$ \\
\hline
\end{tabular}

Table 1: Delivered beam species since 2000.

\section{Beams for COSY operation and experiments}

Since 1996 polarized $\mathrm{H}^{-}$ions have been delivered to the cooler synchrotron COSY [2] at the IKP of the Forschungszentrum Jülich. The layout of the synchrotron facility with its subsystems is described in References [4]. The ion sources are operated at $0.5 \mathrm{~Hz}$ repetition rate with $20 \mathrm{~ms}$ pulse length, which is the maximum useful length for the stripping injection into the synchrotron. The colliding beams source (CBS) [6] provides polarized negatively charged protons or deuterons. The original source has been designed and set in operation by groups from the universities Bonn, Cologne and Erlangen as a colliding beams source in continuous operation [7]. The principle of the source is to collide a pulsed nuclear polarized hydrogen or deuterium beam from a ground state atomic beam source with a pulsed neutral cesium (Cs) beam having a kinetic energy of about $45 \mathrm{keV}[5,8]$. In a charge exchange reaction, taking place in a solenoidal field, negatively charged hydrogen or deuteron ions are created at a potential of 4.5 to $8 \mathrm{kV}$ and accelerated toward the extraction elements. The COSY CBS in a current configuration is described in details in References [10]. The polarization of the circulating beam in COSY is measured routinely during acceleration and at maximum momentum with the internal EDDA detector and exceeds $70 \%$ after successful compensation of depolarizing resonances for highest beam energies [9]. The polarization of the source is optimized with Breit Rabi techniques and a Lambshift polarimeter behind the ion source. High analyzing powers for elastic scattering of the beams on carbon targets enable efficient determination of the nuclear vector polarization behind the cyclotron, which is in the range of 85-92\% of theoretical limits in routine operation. The bar diagram in Figure 1 displays the delivered polarized and unpolarized beam species, protons and deuterons, according to the COSY program, which is annually evaluated and scheduled by an international advisory committee. An increasing amount of beam time is already scheduled to HESR and FAIR activities, as well as for Jülichs srEDM Initiative. Table 1 summarizes the delivered beam species since 2000. The overall, averaged availability of COSY exceeds $92 \%$ with respect to scheduled beam delivery. More than $58 \%$ of experiments demanded polarized beams.

\section{Improved Diagnostics}

After replacement of many components the efforts are focused on improving diagnostic elements for start-up and operation of the polarized ion source's parts. Figure 2 shows a schematic view of the source with its main systems and diagnostic elements. Each part is now equipped with simple networks cameras with mega pixel resolution. The Cs ionizer is monitored for sparks and depositions on the extraction electrodes. This enables early detection of condensed Cs and allows 


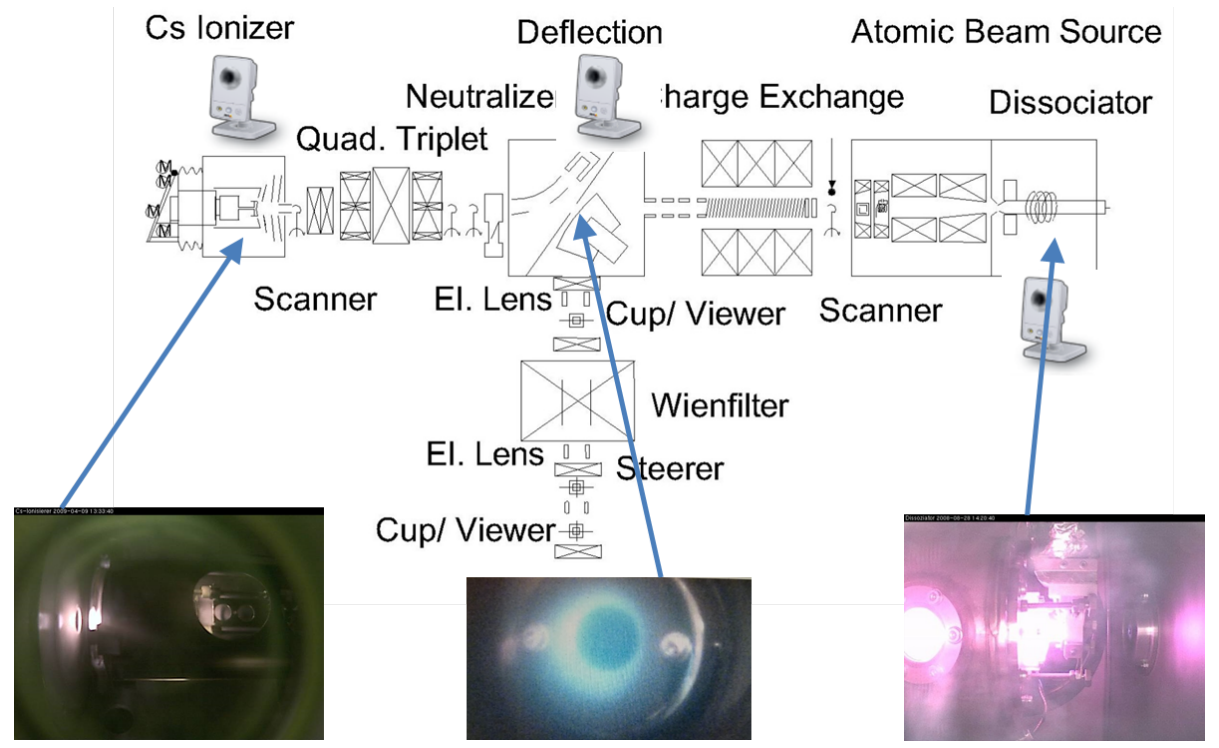

Figure 2: Source diagnostics with network cameras.

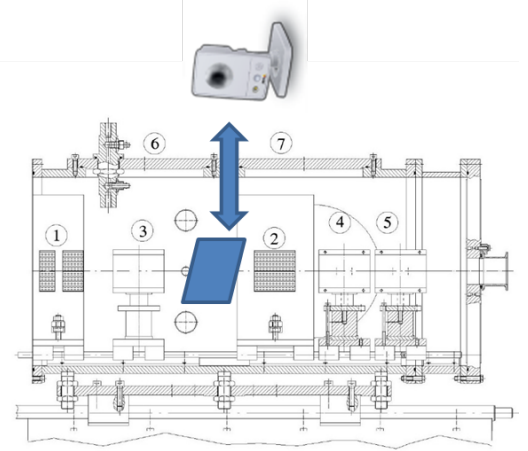

(a) movable viewer plate.

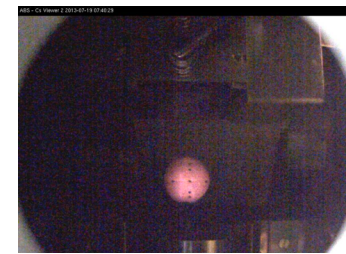

(b) no Cs beam

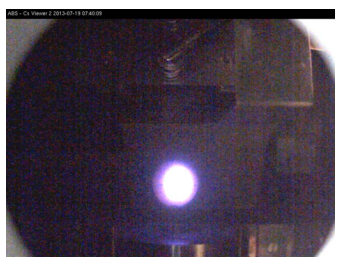

(c) tuned Cs beam

Figure 3: Diagnostics and tuning of pulsed Cs beams.

cleaning before severe sparking occurs. The dissociator is monitored for proper discharge conditions, e.g. discharge color and expansion into the tube. Also deposition of ice at the connection of the tube to the nozzle is easily viewable. Focusing and alignment of the neutral cesium beam with respect to the charge exchange region is under control by a quadrupole triplet, steerer magnets and gun position. The beam is adjusted to a $15 \mathrm{~mm}$ orifice in the deflector chamber before and a movable viewer plate in the atomic beam section behind the solenoid. Figure 3 provides an impression of the beam spot within a radius of $10 \mathrm{~mm}$ inside the atomic beam part. Compared to triggered wire scans of the Cs beam profiles after extraction, quadrupole focusing and after the charge exchange region, the beam is optimized after a few cycles. That has to be compared to a triggered scan, which takes at least two minutes for a full scan with medium resolution at one set point. 


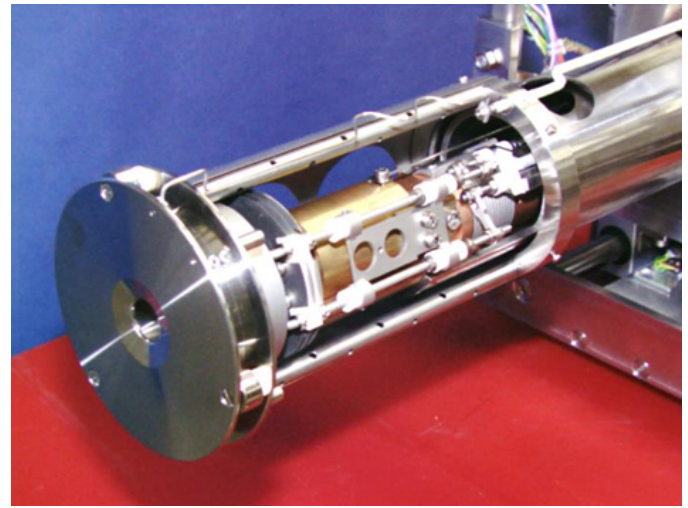

(a) extraction, heater, reservoir and oven.

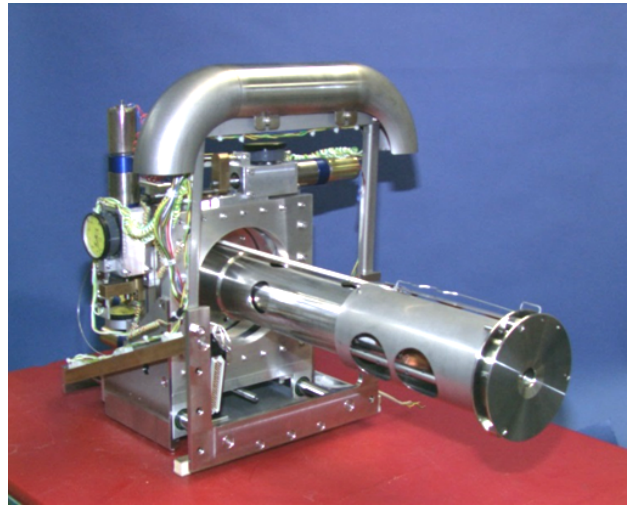

(b) ionizer

Figure 4: Current Cs ionizer set-up, optimized for pulsed operation.

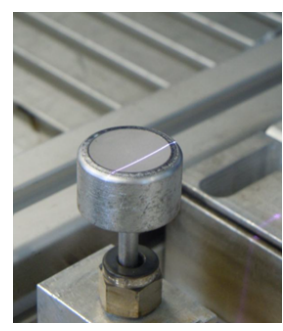

(a) infrared LASER plasma on a dispenser.

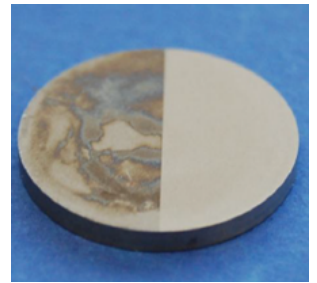

(b) half cleaned dispenser button (20 mm diameter).

Figure 5: LASER treatment of Cs ionizer buttons.

\section{Cesium ionizer dispenser}

The current state of the pulsed cesium ionizer is shown in figure 4. The compact four-axis table allows geometric matching of the extraction system to the performance of the thermal surface ionizer with a porous tungsten dispenser. Electron heating and an optimized cooler/heater module with the cesium reservoir allow a wide range of parameters for optimization. The operational time of the dispenser is limited due to the change of its work function for cesium ionization. By oxidation and sputtering the work function changes and the performance changes slowly with time. During operation this can be compensated by increased dispenser temperature, keeping the emission current constant within small limits. For COSY routine, pulsed operation the increment is about a few watts per week. The ionizer is operational from about 120 to 250 Watt total heating power and provided emission of $6 \mathrm{~mA} /$ peak for more than 2 months. Recently we switch from chemical cleaning procedures, using hazardous acids, to LASER cleaning. Figure 5 depicts the application of a pulsed infrared LASER beam to a used dispenser. The right picture shows a partly cleaned porous tungsten disk. This provides improved handling and welding performance during manufacturing. Also used dispensers with limited performance have been treated. Single treatment of the dispenser surface recovered the work function and surface ionization starts again at low temperatures. A small sample of dispensers had been treated successfully 2 to 3 times up to now. 


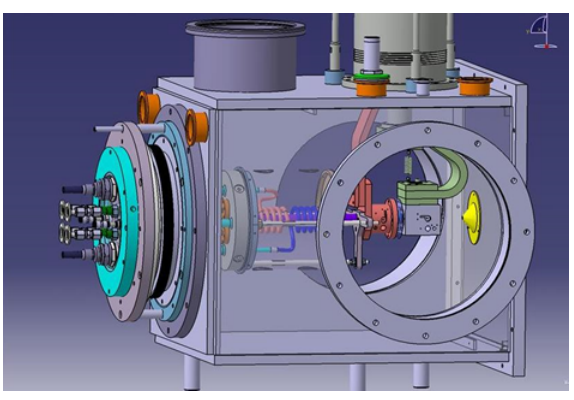

(a) New dissociator setup.

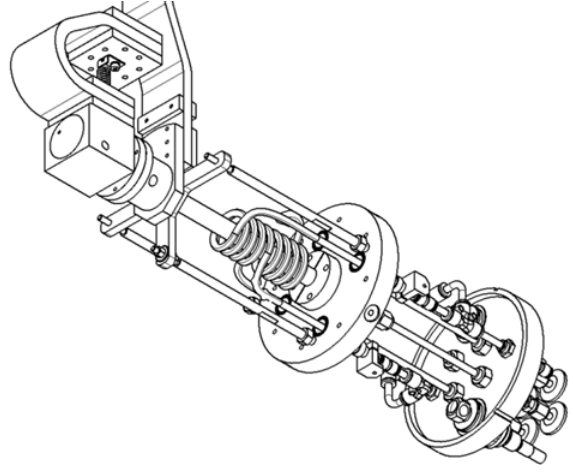

(b) Improved cooling and gas injection.

Figure 6: ABS developments.

\section{Polarized ion beams for COSY and FAIR}

At FZJ the availability of polarized beams with the maximum possible intensity and quality has to be assured. For the planned FAIR project the potential will be enhanced by providing polarized beams. Although polarized beams had not been a prime issue of FAIR at GSI during its inception, their relevance has increased substantially by realizing the potential to address questions that otherwise would be inaccessible. Experimental groups expressed interest in spin physics experiments at the HESR and in the synchrotron design sufficient space is reserved for an upgrade with polarized beams. The research centers of the Helmholtz Association operate accelerator facilities, e.g. at DESY, GSI, HZB, FZD and FZJ, and have an excellent expertise in the field of accelerator physics, together with university partners. The joint Helmholtz program Accelerator Research and Development (ARD)[11] enhances the so far independent research activities, opens up new research opportunities and creates synergies. Official start of the ARD program is in the next funding period 2015-2019. This program will create a national hub for novel accelerator concepts which will develop European and international cooperation in research and education and the IKP receives funding for improving and developing polarized ion sources at COSY and for FAIR. Within that framework the analysis of existing sources and subsystems will be pursued and according to the results a new polarized ion source will be designed and built in the period 2015-2019. The developments will be carried out with international partners and cooperating universities. An ion source, based on the proven concept of resonant charge exchange of a pulsed, nuclear spin-polarized atomic beam with an intense plasma beam, appears as the appropriate approach. A newly designed polarized proton source for FAIR should incorporate techniques and components that have a proven record and have been tested under the demanding routine operation of COSY. The promising experience with atomic beam sources, charge exchange reactions as well as the long-standing successful operation at accelerators at IUCF, Bloomington and at the cooler synchrotron COSY, FZJ and especially the progress at BNL, Brookhaven and INR, Moscow confirm this approach. Pulsed polarized ion sources of INR, Moscow [12] and IUCF, Bloomington [13] produced pulsed polarized atomic hydrogen beams with peak intensities of $2 \times 10^{17} \mathrm{H}^{0} / \mathrm{s}$ and a duration of about $2 \mathrm{~ms}$. The COSY CBS rf discharge dissociator works with a low rf power of $250-450 \mathrm{~W}$ and long pulse duration compared to $2-4 \mathrm{~kW}$ of the INR or the IUCF pulsed sources. 
The active COSY CBS is running with peak intensities of $0.75 \times 10^{17} \mathrm{H}^{0} / \mathrm{s}$ in pulses with $20-100 \mathrm{~ms}$ downstream the hexapole magnets. The new dissociator, figure 6 , with its cooled nozzle is available in a version optimized for higher repetition rate and low gas consumption. In collaboration with the Institute for Nuclear Research (INR, Troitsk, Russia) progress is realized. With the new dissociator and spare hexapoles from an atomic beam target a peak intensity of $1.1 \times 10^{17} \mathrm{H}^{0} / \mathrm{s} \mathrm{has}$ been realized at the test bench [14]. The achievements at the nuclear spin-polarized atomic beam part will be useful to improve COSY operation for the current funding period.

\section{References}

[1] $w w w$.fz-juelich.de Search for IKP, COSY, PAX, JEDI, respectively.

[2] R. Maier Nucl. Instr. Meth. A 390 (1997) P.1.

[3] www.gsi.de/en/research/fair.html.

[4] W. Bräutigam et al.

Proc. of Cyclotrons 1998, Caen, IOP, 1999, P.654;

Proc. of Cyclotrons 2001, East Lansing, AIP 600, 2001, P.123.

[5] W. Haeberli Nucl. Instr. Meth. 62, (1968) P.355.

[6] P.D. Eversheim et al.

Proc. of PST 1994, Köln (1995) P.224;

Proc. of the SPIN 1996 Symposium, Amsterdam.

[7] R. Weidmann et al. Rev. Sc. Instr., Vol. 67 p.II, 1996, P.1357.

[8] M. Eggert et al. NIM A 453, 2000, P.514.

[9] V. Schwarz et al., Proc. SPIN 98, World Sci., 560, (1998).

[10] O. Felden et al.

Proc. of PST 2003, Novosibirsk, RUS NIM A 536 Issue 3, 2005, P.278;

Proc. of 11th EPAC, Edinburgh, GB EPS-AG 2006, P.1705;

Proc. of 11th PNNIB, Santa Fe, USA, AIP 925, 2007, P.105;

Proc. of PSTP 2008, Brookhaven, USA AIP 980, 2008, P.231;

Proc. of PSTP 2009, Ferrara, Italy ISSN 981-4324-91-4, 2009;

Proc. of $2^{\text {nd }}$ Int. Symp. on NIBS, Takayama, Japan, AIP 1390, 2011, P.272.

[11] www.helmholtz-ard.de.

[12] A.S. Belov et al. NIM A 255, 1987, P.442.

[13] V.P. Derenchuk, A.S. Belov Proc. of the PAC 2001, Chicago, USA, (2001) P.2093.

[14] A.S. Belov et al.

IKP Annual Report 2004, Jül 4168 ISSN 0944-2952, 2005, P. 91;

IKP Annual Report 2005, Jül 4212 ISSN 0944-2952, 2006, P. 129;

IKP Annual Report 2006, Jül 4234 ISSN 0944-2952, 2007, P. 131. 